\title{
Determination of Hyperpolarizability Tensor Components by Depolarized Hyper Rayleigh Scattering.
}

\author{
G. J. T. Heesink, A. G. T. Ruiter, N. F. van Hulst, and B. Bölger \\ Department of Applied Physics, University of Twente, P.O. Box 217, 7500 AE Enschede, The Netherlands
}

(Received 4 May 1993)

\begin{abstract}
Depolarized hyper Rayleigh scattering of para-nitroaniline $\left(C_{2 v}\right.$ symmetry $)$ and nitrocalix [4]arene $\left(C_{4}\right.$, symmetry) in solution has been measured. Using linearly and circularly polarized fundamental radiation information about the ratios between the several hyperpolarizability tensor components, including their sign, was obtained. Results are consistent with the theory developed for both symmetry groups. Comparison between experimental depolarization ratios and ratios obtained from $a b$ initio calculated hyperpolarizability tensor components shows good agreement.
\end{abstract}

PACS numbers: $42.65 . \mathrm{Ky}$

Hyper Rayleigh scattering (HRS) allows molecular hyperpolarizabilities $\beta$ in solution to be obtained in a direct and accurate manner as the HRS signal is directly proportional to $\beta^{2}$ and the solvent acts as an internal reference $[1-3]$. With respect to this HRS has some advantages over the more commonly used technique of electric field induced second harmonic generation (EFISHG) [4,5]. The latter method implies alignment of molecules in a solvent by their dipole moment $\mu$ by applying a static electric field. Because of the alignment only the projection component of the molecular hyperpolarizability tensor along the dipole moment can be measured. In fact one measures $\gamma+\mu \beta / 5 k T$, where $\gamma$ is the second order hyperpolarizability. Therefore knowledge about $\gamma$ and $\mu$ is needed to extract the hyperpolarizability $\beta$ from EFISHG measurements. HRS requires no alignment of the molecules or knowledge of their dipole moment. As a consequence HRS in contrast with the EFISHG technique also applies to nonpolar molecules, e.g., $\beta$ of octupolar origin [6,7], ionic species, and solutions with a polar solvent. Comparison of HRS and EFISHG data enables determination of the angle between the molecular dipole moment $\mu$ and the vectorial component of the $\beta$ tensor.

The HRS signal is proportional to an orientational average over the square of a combination of hyperpolarizability tensor components. Use of different orientations of polarized light and analysis of the degree of polarization of the HRS signal allows deconvolution of the orientational average and thus separation of the $\beta$ tensor components for molecules of low symmetry [8,9]. Apart from the initial work of Terhune [10] and Maker [11] this quality of depolarized HRS has not yet been investigated experimentally.

The features of depolarized HRS are shown in this Letter for para-nitroaniline ( $p \mathrm{NA})$, as the molecule is one of the best documented donor-acceptor systems, and for nitrocalix[4]arene. Calix[4] arenes are a new class of nonlinear optical compounds with a unique combination of four nonconjugated $D-\pi-A$ dipoles in one molecule [12]. These compounds show an enhanced hyperpolarizability which is not accompanied by the usual redshift of the charge transfer band. The nitrocalix [4] arene mole- cule investigated contains four $\mathrm{NO}_{2}$ groups in cone conformation. Corona poled thin films of nitrocalix[4] arenes combine high $\chi^{(2)}$ values with good temporal stability, making these films suitable for efficient second harmonic generation [13].

The induced dipole moment of a molecule in the orthogonal molecular fixed coordinate system $1,2,3(\Longleftrightarrow i$, $j, k)$ is given by $\mu_{i}=\sum_{j} \alpha_{i j}+\sum_{j, k} \beta_{i j k} E_{j} E_{k}+\cdots$, where $\mu_{i}$ is the dipole moment along the $i$ axis, $\alpha_{i j}$ is the $i j$ component of the polarizability tensor $\alpha, \beta_{i j k}$ is the $i j k$ component of the hyperpolarizability tensor $\beta$, and $E_{j}$ is the electric field component along the $j$ axis. HRS depends on the third rank hyperpolarizability tensor $\beta$ in the expansion of the induced dipole moment of the molecule. Considering only the induced dipole at frequency $2 \omega$ in the space fixed orthogonal coordinate system $x, y$, $z(\Longleftrightarrow u, v, w)$ the field intensity $I_{w}(2 \omega)$ for polarization in the $w=x$ or $z$ direction and propagation in the $y$ direction (Fig. 1) is

$$
I_{w}(2 \omega)=\mathcal{C}\left\langle\mu_{w}^{2}(2 \omega)\right\rangle_{t},
$$

where $\mathcal{C}=(2 \omega)^{4} / 16 \pi^{2} \varepsilon_{0} c^{3}$, the subscript $t$ refers to a time average over the period of incident radiation, and $\left\langle\mu_{w}^{2}(2 \omega)\right\rangle=\int_{\Omega} d \Omega \mu_{w}^{2}(2 \omega)$, where $\Omega$ denotes the Euler angles of the molecule. For molecules which contribute incoherently to the total observed HRS signal the average over the total number of molecules of the time averaged $\beta$ component is calculated by multiplying the $\beta$ component

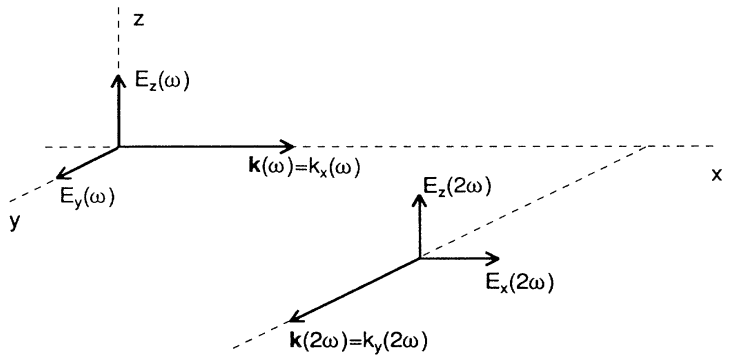

FIG. 1. Propagation and polarization directions of the fundamental and second harmonic radiation field. 
of a single molecule by the total number of molecules. In that case the intensity of the HRS signal for an $n$ component solution and fundamental radiation propagating in the $x$ direction, with an electric field $\mathbf{E}(\omega)$ $=E_{y}(\omega) \mathbf{e}_{y}+E_{z}(\omega) \mathbf{e}_{z}$ (Fig. 1), is given by

$$
\begin{aligned}
I_{w}(2 \omega)= & e f^{4}(\omega) f^{2}(2 \omega) E_{0}^{4}(\omega) \\
& \times \sum_{n} C_{n}\left[\sum_{u v} \sum_{u^{\prime} v^{\prime}} E_{u} E_{v} E_{u^{\prime}} E_{v^{\prime}}\left\langle\beta_{w u v} \beta_{w u^{\prime} v^{\prime}}\right\rangle_{n}\right],
\end{aligned}
$$

where $w=x$ or $z, f(\omega)$ and $f(2 \omega)$ are the local field factors at the fundamental and second harmonic frequency, respectively, $E_{0}^{2}(\omega)=E_{u}^{2}(\omega)+E_{v}^{2}(\omega), \quad E_{u, v}=E_{u, v}(\omega) /$ $E_{0}(\omega)$, and $C_{n}$ is the concentration of solute $n$. The quantity $\sum_{u v} \sum_{u^{\prime} v^{\prime}} E_{u} E_{v} E_{u^{\prime}} E_{v^{\prime}}\left\langle\beta_{w u v} \beta_{w u^{\prime} v^{\prime}}\right\rangle$ is now referred to as $\beta_{w, \mathrm{HRS}}^{2}$, which is dominated by the $\beta$ component in the direction of the molecular symmetry axis (3-axis) for $\pi$ conjugated systems. The dependence of $\beta_{\text {HRS }}$ on the polarity of the solvent with dielectric constant $\varepsilon$ is shown to be in good approximation proportional to $(\varepsilon-1) /(2 \varepsilon$ $+1)$, which expresses the relation to the reaction field experienced by the permanent dipole moment of the solute molecule due to its interaction with the permanent dipole moments of the solvent molecules [1]. A more specific expression for Eq. (2) is obtained by expressing the incident fundamental electric field in parameters of ellipticity and phase,

$$
\mathbf{E}(\omega)=E_{0}\left[\cos \Psi \cos (\omega t) \mathbf{e}_{y}+\sin \Psi \cos (\omega t+\delta) \mathbf{e}_{z}\right],
$$

in which $\delta$ is the phase and $\Psi$ the angle of the principal elliptic axis with $\mathbf{e}_{y}$. It is calculated for detection in the $y$ direction [8],

$$
\begin{aligned}
I_{w}(2 \omega)=e f^{4}(\omega) f^{2}(2 \omega) E_{0}^{4}(\omega) \sum_{n} C_{n}[ & \left\langle\beta_{w y y}^{2}\right\rangle \cos ^{4} \Psi+\left\langle\beta_{w z z}^{2}\right\rangle \sin ^{4} \Psi \\
& \left.+\left\langle\left(\beta_{w y z}+\beta_{w z y}\right)^{2}+2 \beta_{w y y} \beta_{w z z} \cos (2 \delta)\right\rangle \sin ^{2} \Psi \cos ^{2} \Psi\right]_{n}
\end{aligned}
$$

Measurement of $I_{x}(2 \omega)$ and $I_{z}(2 \omega)$ for different values of $\Psi$ and $\delta$ gives specific information on the relation between the several tensor components. In fact, up to five independent observables can be determined by using adequate polarization directions [8]. The average $\left\langle\beta_{w u v} \beta_{w u^{\prime} v^{\prime}}\right\rangle$ over all molecular directions has been calculated by Bersohn [8] for linearly polarized incident radiation for several high symmetry point groups including the $C_{2 v}$, point group which applies to $p$ NA. As to the case of nitrocalix[4]arene molecules with $C_{4 v}$, symmetry the number of independent tensor components is reduced to two, $\beta_{311}=\beta_{131}=\beta_{113}=\beta_{223}=\beta_{232}=\beta_{322}$ and $\beta_{333}$, where Kleinman symmetry has been assumed which is reasonable at $532 \mathrm{~nm}$ for the absorption maximum of the nitrocalix [4] arene is at $\lambda_{\max }=291 \mathrm{~nm}[12,13]$. A coordinate transformation between space fixed and molecular fixed coordinates involving the averaging of all the products of the direction cosines over all directions gives

$$
\begin{gathered}
\left\langle\beta_{z z z}^{2}\right\rangle=\frac{3}{105} \beta_{333}^{2}\left[5+12\left(\beta_{311} / \beta_{333}\right)+24\left(\beta_{311} / \beta_{333}\right)^{2}\right] \\
\left\langle\beta_{x z z}^{2}\right\rangle=\frac{1}{105} \beta_{333}^{2}\left[3-4\left(\beta_{311} / \beta_{333}\right)+20\left(\beta_{311} / \beta_{333}\right)^{2}\right] \\
\left\langle\beta_{x y y} \beta_{x z z}\right\rangle=\frac{1}{105} \beta_{333}^{2}\left[1+8\left(\beta_{311} / \beta_{333}\right)+2\left(\beta_{311} / \beta_{333}\right)^{2}\right] \\
\left\langle\beta_{z y y} \beta_{z z z}\right\rangle=\frac{3}{105} \beta_{333}^{2}\left[1+8\left(\beta_{311} / \beta_{333}\right)+2\left(\beta_{311} / \beta_{333}\right)^{2}\right] \\
\left\langle\left(\beta_{z y z}+\beta_{z z y}\right)^{2}\right\rangle=\frac{4}{105} \beta_{333}^{2}\left[3-4\left(\beta_{311} / \beta_{333}\right)\right. \\
\left.+20\left(\beta_{311} / \beta_{333}\right)^{2}\right] \\
\left\langle\left(\beta_{x y z}+\beta_{x z y}\right)^{2}\right\rangle=\frac{4}{105} \beta_{333}^{2}\left[1-6\left(\beta_{311} / \beta_{333}\right)\right. \\
\left.+9\left(\beta_{311} / \beta_{333}\right)^{2}\right] .
\end{gathered}
$$

Considering an isotropic solution, for which $\left\langle\beta_{x y y}^{2}\right\rangle$ $=\left\langle\beta_{z y y}^{2}\right\rangle=\left\langle\beta_{x z z}^{2}\right\rangle$, and then by using linearly and circularly polarized fundamental radiation, detection of the two
HRS polarization allows determination of three independent depolarization ratios, $D_{z z}^{z x}=I_{z, x}(2 \omega) / I_{z, z}(2 \omega), D_{c z}^{c x}$ $=I_{c, x}(2 \omega) / I_{c, z}(2 \omega)$, and $D_{z z}^{c z}=I_{c, z}(2 \omega) / I_{z, z}(2 \omega)$. The first and second subscripts refer to the polarization state of incident and detected radiation, respectively. The subscript $c$ denotes circularly polarized radiation. In case of Kleinman symmetry this reduces to only two independent depolarization ratios, $D_{c z}^{c x}$ and $D_{z z}^{c z}=2 D_{z z}^{z x}$.

In Fig. 2, the calculated depolarization ratios for $C_{4 i}$ symmetry are given as a function of $\beta_{311} / \beta_{333}$. For $\beta_{311}=0$, i.e., a pure vectorial $\beta$ along $\mu$, the depolarization ratios are $D_{z z}^{z x}=0.20, D_{c z}^{c x}=0.33$, and $D_{z z}^{c z}=0.40$ for $z$ linearly polarized and circularly polarized incident radiation. For a positive $\beta_{311} / \beta_{333}$ ratio the HRS signal is increasingly polarized with the highest polarization degree for $\beta_{311}=\beta_{333} / 3$. For the latter situation the HRS signal for circularly polarized radiation is completely polarized along the $z$ direction. For a negative $\beta_{311} / \beta_{333}$ ratio the HRS signal is less polarized with the depolarization ratio closest to unity at $\beta_{311}=-\beta_{333} / 2$. It should be noted that, as is clear from Fig. 2, depolarized HRS enables determination of the relative sign of the $\beta$ tensor components.

The experimental HRS setup consists of a $4 \mathrm{~cm}$ long cell containing $3 \mathrm{ml}$ of the solution under investigation. Fundamental radiation from a Nd:YAG laser is focused by a $150 \mathrm{~mm}$ lens into the cell. Incident power and polarization $(\Psi, \delta)$ are adjusted by a set of half and quarter wave plates in combination with a Glan-Taylor polarizer. A photodiode is used to monitor the intensity of the fundamental beam. The HRS signal is collected perpendicular to the fundamental beam by a suitable condenser system with reduced NA $(\cong 0.15)$ in order to avoid signal averaging over directions significantly deviating from $\mathbf{e}_{y}$. 


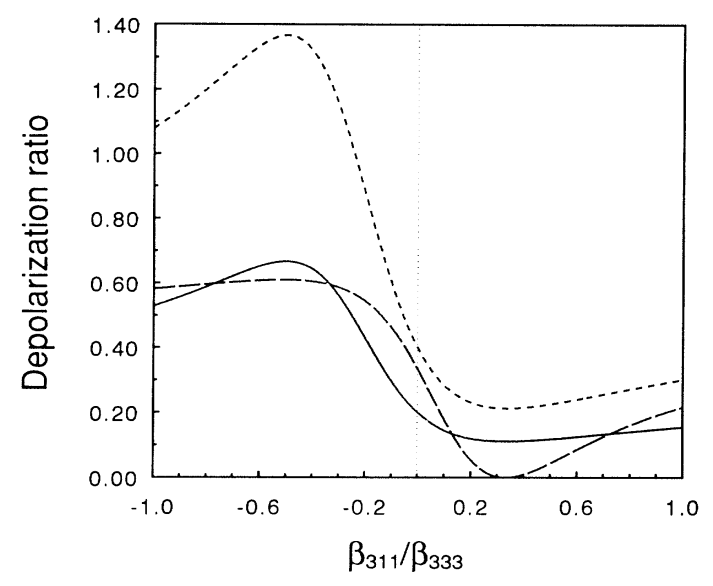

FIG. 2. Theoretical depolarization ratios as a function of $\beta_{311} / \beta_{333}$ for molecules with $C_{4 v}$ symmetry. (—: $D_{z z}^{z x},--$ : $D_{c z}^{c x}, \cdots: D_{z z}^{c z}$.)

Separation of scattered fundamental and the second harmonic radiation is achieved using a $532 \mathrm{~nm}$ interference filter with a $2.6 \mathrm{~nm}$ bandwidth. This bandwidth is narrow enough to exclude detection of possible hyper Raman contributions which has been verified experimentally. To extract information about the polarization state of the HRS signal a rotatable analyzer plate is mounted between the cell and a photomultiplier tube. The signal is fed into a boxcar averager in combination with a PC for data acquisition.

Measurements of the depolarized HRS signal intensity for $p \mathrm{NA}$ in chloroform are shown in Fig. 3 as a function of the analyzer angle for incident $y$ and $z$ linearly and circularly polarized radiation. Both the constant value of $I(2 \omega)$ for $y$ linearly polarized incident radiation and its coincidence with $I_{z, x}(2 \omega)$ are due to the isotropy of the solution. Depolarization ratios calculated from these measurements are $D_{z z}^{z x}=0.230(0.013), D_{c z}^{c x}=0.39(0.03)$, and $D_{z z}^{c z}=0.49(0.04)$. For $p N A$ in other solvents, methanol and dioxane, the same depolarization ratios are found within experimental accuracy. Therefore it is concluded that the solvent effect as to the depolarization ratios is negligible. Measurements for different concentrations of $p$ NA in the various solvents showed the depolarization ratio to be independent of the concentration. Results are shown in Table I together with depolarization ratios which were calculated from theoretical $a b$ initio $\beta$ values. For $C_{2 v}$, symmetric $p \mathrm{NA}$ theoretical expressions for the depolarization ratios were calculated for $z$ linear [8] and circular polarization in a manner similar to that discussed for the $C_{4}$, symmetry. Experimental results are in excellent agreement with the depolarization ratios obtained from the $\beta$ values of Lalama and Garito [14] who calculated five independent $\beta$ tensor components at $\lambda=1064 \mathrm{~nm}$, not assuming Kleinman symmetry, and the Møller-Plesset (MP2) based static $(\omega=0)$ model of Sim

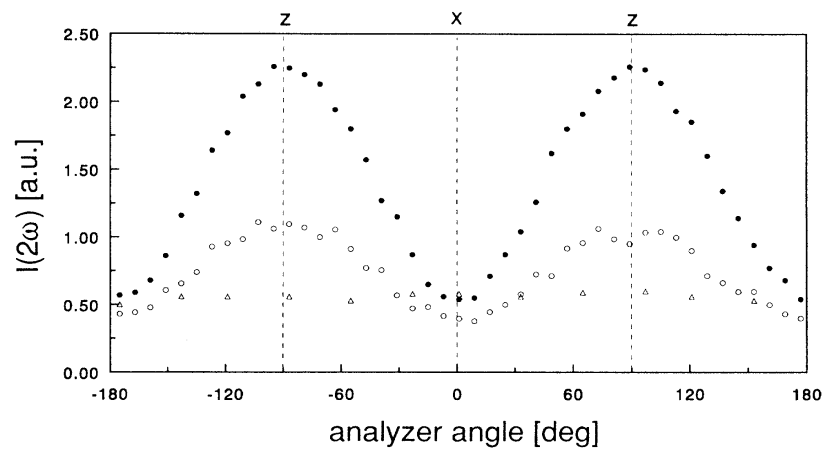

FIG. 3. HRS signal as a function of the analyzer angle for $p$ NA $(20 \mathrm{mmol} / \mathrm{l}$ in chloroform) for different states of incident polarization [०: $\left.I_{z}(\vartheta), \bigcirc: I_{c}(\vartheta), \Delta: I_{y}(\vartheta)\right]$.

and Rice [15] who impose Kleinman symmetry which yields three independent $\beta$ values. Depolarization ratio values obtained from the Hartree-Fock (HF) based model of Sim and Rice, the finite field (FF) and the sum over states (SOS) models of Velders [16], and the Austin finite field model (AM1) and the coupled perturbed Hartree-Fock (CPHF 3-21 G) models of Brédas [17] are considerably higher due to overestimated nonvectorial $\beta$ components. Owing to underestimation of the latter $\beta$ components the intermediate neglect of differential overlap (INDO) model of Brédas yields depolarization ratios that are too low.

HRS measurements on $p$ NA solutions show quadratic dependence on the fundamental intensity. For $p$ NA in chloroform it was found by the internal reference method (IRM) [1], $\beta_{\mathrm{HRS}, p N A}=32(3) \times 10^{-30} \mathrm{esu}$, with $\beta_{\mathrm{CHCl}_{3}}$ $=-0.49(0.05) \times 10^{-30}$ esu [18] as the internal reference. Teng and Garito [19] and Oudar and Chemla [20]

TABLE I. Theoretical and experimental depolarization ratios for $p$ NA.

\begin{tabular}{lccc}
\hline \hline & $D_{z z}^{z x}$ & $D_{c z}^{c x}$ & $D_{z z}^{c z}$ \\
\hline Solvent & & Experimental \\
Chloroform & $0.230 \pm 0.013$ & $0.39 \pm 0.03$ & $0.49 \pm 0.04$ \\
Methanol & $0.225 \pm 0.011$ & $0.37 \pm 0.03$ & $0.46 \pm 0.03$ \\
Dioxane & $0.225 \pm 0.011$ & $0.39 \pm 0.03$ & $0.48 \pm 0.04$ \\
& & Theoretical & \\
Theor. model & & & \\
[14] & & 0.407 & 0.526 \\
[15] HF & 0.236 & 0.466 & 0.588 \\
$\quad$ MP2 & 0.294 & 0.379 & 0.450 \\
[16] FF & 0.225 & 0.460 & 0.576 \\
$\quad$ SOS & 0.288 & 0.513 & 0.748 \\
[17] AM1 & 0.374 & 0.458 & 0.570 \\
$\quad$ 3-21G & 0.290 & 0.463 & 0.580 \\
INDO & 0.204 & 0.339 & 0.408 \\
\hline \hline
\end{tabular}


found by the EFISHG method $\beta_{p \mathrm{NA}}=16.9(0.4) \times 10^{-30}$ esu in dioxane and $\beta_{p N A}=34.5(4.0) \times 10^{-30}$ esu in methanol, respectively. Taking into consideration the vectorial character of the hyperpolarizability $\beta$ as obtained by the EFISHG method [for $p$ NA $\beta_{3}=\beta_{333}$ $+\frac{1}{3}\left(\beta_{322}+2 \beta_{223}\right)$, with $\beta_{322} / \beta_{333} \cong-0.154$ and $\beta_{223} / \beta_{333}$ $\cong-0.09$ [14]] it was calculated $\beta_{\mathrm{HRS}, p \mathrm{NA}}=19(1)$ $\times 10^{-30} \mathrm{esu}$ in dioxane and $\beta_{\mathrm{HRS}, p \text { NA }}=40(5) \times 10^{-30} \mathrm{esu}$ in methanol. Owing to the linear dependence of the hyperpolarizability $\beta_{\text {HRS }}$ as to the solvent related factor $(\varepsilon-1) /(2 \varepsilon+1) \quad(0.22,0.48$, and 0.36 for dioxane, methanol, and chloroform, respectively) it was concluded that the $\beta_{\mathrm{HRS}, p \text { NA }}$ value in chloroform as was measured is in good agreement with the $\beta_{p N A}$ values in dioxane and methanol as were obtained by the EFISHG method. The value $\beta_{\mathrm{HRS}, p \text { NA }}=23(3) \times 10^{-30}$ esu found by Clays [1] is somewhat lower.

Measurement of the depolarized HRS signal of the nitrocalix [4] arene gives

$$
D_{z z}^{z x}=0.21(0.01), \quad D_{c z}^{c x}=0.35(0.02),
$$

and

$$
D_{z z}^{c z}=0.39(0.03) \text {. }
$$

These experimental values are in their combination consistent with the theory for the $C_{4 v}$ symmetry. The result implies that $\left|\beta_{311}\right| \leq 0.02\left|\beta_{333}\right|$ and the ratio $\beta_{311} / \beta_{333}$ is negative. As to this result it has to be concluded that for very small off-diagonal $\beta$ coefficients the absolute accuracy of the measurements is of considerable importance to the value and sign of the ratio $\beta_{311} / \beta_{333}$; therefore improvement of the accuracy in the results is appropriate.

For the nitrocalix [4] arene a value of $\beta_{\mathrm{HRS} \text {, calix }}=28$ (3) $\times 10^{-10} \mathrm{esu}$ in chloroform is found by the IRM method which is in agreement with literature [21]. To obtain reproducible results it was necessary to use moderate laser power and to renew the nitrocalix [4] arene solutions between the several measurements in order to reduce the fluorescence signal which originated from nitrocalix[4] arene molecules which were degenerated by high laser power.

It is concluded that depolarization measurements on the HRS signal give information about the ratios between the several hyperpolarizability tensor components including their sign. Knowledge of the several independent hyperpolarizability tensor components may be of particular interest in the case of nondipolar molecules, such as octupolar molecules. Absolute values can be obtained in the case of molecules with enough symmetry if this technique is combined with the IRM. Further investigation as to the angular dependence of the HRS signal is appropriate. Depolarized HRS is a direct test for the validity of Kleinman symmetry. The experimental results of the depolari- zation measurements are a suitable probe for $a b$ initio calculated hyperpolarizability components.

This research was supported by the Dutch Foundation for Fundamental Material Research (FOM) and the Dutch Ministry of Finances (IOP BP102). We thank Dr. E. Kelderman for making available the calix [4]arenes and Dr. C. M. J. Wijers for valuable discussions.

[1] K. Clays and A. Persoons, Phys. Rev. Lett. 66, 2980 (1991).

[2] K. Clays and A. Persoons, Rev. Sci. Instrum. 63, 3285 (1992).

[3] K. Clays and A. Persoons, Adv. Chem. Phys. (to be published).

[4] C. G. Bethea, Appl. Opt. 14, 1447 (1975).

[5] G. R. Meredith, Rev. Sci. Instrum. 53, 48 (1982).

[6] J. Zyss, Nonlinear Opt. 1, 3 (1991).

[7] T. Verbiest, K. Clays, and A. Persoons, Opt. Lett. 18, 525 (1993).

[8] R. Bersohn, Y.-H. Pao, and H. L. Frisch, J. Chem. Phys. 45, 3184 (1965) (the factor for the $s_{24}$ coefficient in the expansion of $\left\langle\beta_{x z z}^{2}\right\rangle$ in appendix $B$ for the $C_{2 v}$ point group should be $\left.\frac{2}{105}\right)$.

[9] S. J. Cyvin, J. E. Rauch, and J. C. Decius, J. Chem. Phys. 43, 4083 (1965).

[10] R. M. Terhune, P. D. Maker, and C. M. Savage, Phys. Rev. Lett. 14, 681 (1965).

[11] P. D. Maker, Phys. Rev. A 1, 923 (1970).

[12] E. Kelderman, L. Derhaeg, G. J. T. Heesink, W. Verboom, J. F. J. Engbersen, N. F. van Hulst, A. Persoons, and D. N. Reinhoudt, Angew. Chem. 31, 1075 (1992).

[13] G. J. T. Heesink, N. F. Van Hulst, B. Bölger, E. Kelderman, J. F. J. Engbersen, W. Verboom, and D. N. Reinhoudt, Appl. Phys. Lett. 62, 2015 (1993).

[14] S. J. Lalama and A. F. Garito, Phys. Rev. A 20, 1179 (1979).

[15] F. Sim, S. Chin, M. Dupuis, and J. E. Rice, J. Chem. Phys. 97, 1158 (1993).

[16] G. J. M. Velders, J.-M. Gillet, P. J. Becker, and D. Feil, J. Phys. Chem. 95, 8601 (1991).

[17] J. L. Brédas and F. Meyers, J. Am. Chem. Soc. 114, 4927 (1992)

[18] F. Kazjar, I. Ledoux, and J. Zyss, Phys. Rev. A 36, 2210 (1987).

[19] C. C. Teng and A. F. Garito, Phys. Rev. B 28, 6766 (1983).

[20] J. L. Oudar and D. S. Chemla, J. Chem. Phys. 66, 2664 (1977).

[21] T. Verbiest, L. Derhaeg, E. Kelderman, J. F. J. Engbersen, W. Verboom, D. N. Reinhoudt, K. Clays, and A. Persoons, in Proceedings of the Third International Symposium on Organic Materials for Nonlinear Optics, 19-21 August 1992, University of Oxford (to be published). 